Einführung zum Thema

Orthopäde $2020 \cdot 49: 750$

https://doi.org/10.1007/s00132-020-03951-3

(c) Springer Medizin Verlag GmbH, ein Teil von Springer Nature 2020

Liebe Leserinnen und Leser,

Revisionseingriffe sind für den operativ tätigen Behandler eine Herausforderung und für den Patienten eine hoffnungsvolle Aussicht auf Funktionsverbesserung. Hierbei ist das $\mathrm{zu}$ erwartende postoperative Ergebnis meist schwieriger einzuschätzen als beim Primäreingriff. Aus diesem Grunde sind die genaue Analyse des Versagens der Primäroperation, Indikation zur Revision und die operative Durchführung äußerst anspruchsvoll und wesentlich von der klinischen Erfahrung des Operateurs abhängig.

Die häufigsten Nervenkompressionssyndrome, das Karpaltunnelsyndrom und das Kubitaltunnelsyndrom (,Sulcus-ulnaris-Syndrom"), werden überwiegend operativ therapiert und zeigen meist sehr gute postoperative Ergebnisse. Revisionseingriffe können bei persistierenden Beschwerden indiziert sein, setzen spätestens dann aber eine mikrochirurgische Technik voraus. In Zusammenarbeit mit den Wiener Kollegen vom Millesi Center haben die Herausgeber aus Bad Rappenau eine Übersicht zu Revisionen bei diesen beiden Nervenkompressionssyndromen zusammengestellt.

Die Rhizarthrose gehört zu den häufigsten arthrotischen Erkrankungen. Die Resektionsarthroplastik als Standardeingriff erzielt in der überwiegenden Zahl der Fälle sehr gute Ergebnisse. Beschwerdepersistenzen oder gar Verschlechterungen nach operativer Therapie können allerdings verbleiben und stellen eine diagnostische und operative Herausforderung dar. Steffen Löw stellt mit seinen Koautoren ausführlich

\author{
Christian K. Spies ${ }^{1}$ Frank Unglaub ${ }^{1,2}$ \\ ${ }^{1}$ Handchirurgie, Vulpius Klinik, Bad Rappenau, Deutschland \\ ${ }^{2}$ Medizinische Fakultät Mannheim, Universität Heidelberg, Mannheim, Deutschland
}

\title{
Revisionseingriffe an der Hand
}

die Komplexität der Diagnostik und Therapiemöglichkeiten vor.

Verklebungen von Sehnen können nach Ringbandspaltungen, aber auch nach Sehnennähten auftreten. Bernd Hohendorff und Mitautoren geben eine breite Übersicht zu konservativen und operativen Therapieverfahren.

Bewegungserhaltende Handgelenkeingriffe wie Teilarthrodesen oder Resektionsarthroplastiken sind zentrale Bausteine zur Therapie zahlreicher Handgelenkpathologien. Die Entwicklung von modernen Osteosynthesematerialien mit anatomisch geformtem Plattendesign und winkelstabiler Verblockung bieten im Vergleich zu K-Drahtosteosynthesen in der Regel eine stabilere Versorgung. Dennoch kann dadurch meist nicht auf eine Knochentransplantation verzichtet werden. Die Herausgeber und Mitautoren dieses Themenhefts beleuchten diesbezüglich die Revisionen der häufigsten (Teil-)Versteifungen näher.

Die Handgelenkendoprothetik ist ein noch relativ junger Bereich in der Handchirurgie. Mit den Neuentwicklungen auf diesem Gebiet verlängern sich auch die Standzeiten der Prothesen. Da die Anzahl der implantierten Endoprothesen am Handgelenk stetig steigt, gehen Hans-Georg Damert und seine Kollegen auf die Revisionen in diesem Gebiet ein.

Liebe Leserinnen und Leser, wir möchten in diesem Leitthemenheft „Revisionseingriffe an der Hand" hinsichtlich Indikationsstellung, operativer Durchführung und Nachsorge für häufige Hand- und Handgelenkeingriffe sensibilisieren. Wir hoffen gleichzeitig auch, durch die langjährige Erfahrung der Autoren Tipps und
Tricks für den Primäreingriff geben zu können, um die Anzahl an Revisionseingriffe an der Hand so niedrig wie möglich zu halten.

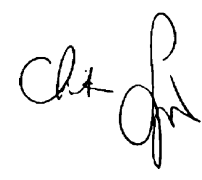

PD Dr. Christian K. Spies

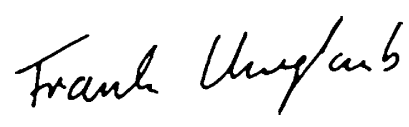

Prof. Dr. Frank Unglaub

\section{Korrespondenzadresse}

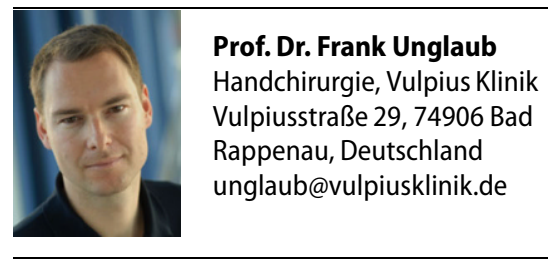

Interessenkonflikt. C.K. Spies und F. Unglaub halten regelmäßig Vorträge für die IBRA, Basel, Schweiz. 